\title{
An Asymptotic Formula for a Class of Distribution Functions.
}

By E. W. Cannon and Adrel Wintwer, Johns Hopkins University. Communicated by A. C. Aitken.

(Received 25th February, 1935. Read 1st March, 1935.)

If $x_{1}, x_{2}, \ldots, x_{k}, \ldots$ are independent random variables each of which is subjected to a distribution law $\sigma=\sigma(x)$ independent of $k$ and having a finite positive dispersion, then $x_{1}+x_{2}+\ldots+x_{n}$ is known to obey the Gauss law as $n \rightarrow+\infty$, no matter how $\sigma(x)$ be chosen 1 . There arises, however, the question whether it is nevertheless possible to determine the elementary law $\sigma(x)$ from the asymptotic behaviour of the distribution law of $x_{1}+x_{2}+\ldots+x_{n}$ for very large but finite values of $n$. It will be shown that the answer is affirmative under very general conditions.

Let the distribution function $\sigma(x)$ be a solution of the moment problem

$$
\int_{-\infty}^{+\infty} x^{m} d \sigma(x)=M_{m} \quad(m=0,1,2, \ldots ; \sigma(-\infty)=0),
$$

so that $M_{0}$ is the total probability, hence equal to 1 . It is not required that (1) be a determined moment problem, i.e. that $\sigma$ be uniquely determined by the conditions (1) if one normalises it by the requirement that $2 \sigma(x)=\sigma(x+0)+\sigma(x-0)$. On excluding the case $M_{2}=0$ of the trivial distribution function $\sigma(x)=\frac{1}{2}(1+\operatorname{sign} x)$ and replacing, if necessary, $\sigma(x)$ by $\sigma(a x)$, where $a=M_{2}^{\frac{1}{2}}>0$, we may suppose that $M_{2}=1$. Also, although the symmetry condition thereby imposed upon the law $\sigma$ is not essential for our method, we assume for convenience that both ranges $(0, x)$ and $(-x, 0)$ of the random variable subjected to $\sigma$ are equally probable, i.e. that

(2) $\sigma(x)+\sigma(-x)=1$; hence $\sigma(0)=\frac{1}{2}, M_{2 n+1}=0 \quad(n=0,1,2, \ldots)$.

Accordingly, the characteristic function of $\sigma$,

$$
L(t ; \sigma)=\int_{-\infty}^{+\infty} e^{i t x} d \sigma(x) \quad(-\infty<t<+\infty),
$$

1 Cf. P. Lévy, Calcul des Probabilités, Paris, 1925, pp. 233-235. 
and its Fourier inversion ${ }^{1}$,

$$
\sigma(x)=\sigma(0)-\frac{1}{2 \pi i} \int_{-\infty}^{+\infty} L(t ; \sigma)\left(e^{-i x t}-1\right) t^{-1} d t \quad(-\infty<x<+\infty),
$$

become respectively

and

$$
L(t ; \sigma)=2 \int_{0}^{+\infty} \cos (t x) d \sigma(x) \quad(-\infty<t<+\infty)
$$

$$
\sigma(x)=\frac{1}{2}+\frac{1}{\pi} \int_{0}^{+\infty} L(t ; \sigma) t^{-1} \sin (t x) d t .
$$

We finally suppose that for some sufficiently small $\delta>0$ and for some function $\phi(t)$

$$
\int^{+\infty}\left\{t \phi(t)^{1 / \delta}\right\}^{-1} d t<+\infty \text {, and } L(t ; \sigma)=O(1 / \phi(t)) \text { as } t \rightarrow+\infty,
$$

consideration of $t \rightarrow-\infty$ being unnecessary since $L(t ; \sigma)$ is an even function, and that

$$
L(t ; \sigma) \rightarrow 0 \text { as } t \rightarrow \infty .
$$

A few remarks concerning the nature of the restriction imposed by conditions (5) and (5a) upon the behaviour of $\sigma(x)$ are not out of place. According to Lévy ${ }^{2}$ the average of $|L(t ; \sigma)|^{2}$ in the whole range $-\infty<t<+\infty$ always exists and is equal to the sum of the squares of all jumps of $\sigma(x)$. Hence $\sigma(x)$ is everywhere continuous if and only if the average of $|L(t ; \sigma)|^{2}$ is zero, a condition clearly satisfied whenever $(5 a)$ is satisfied, so that $\sigma$ has no discontinuity points. However (5), (5a) are sufficiently general not to require the absolute continuity of $\sigma$, i.e. the existence of a density of probability ${ }^{3}$. In fact (5) and (5a) are implied by

$$
L(t ; \sigma)=O\left(|\log t|^{-\alpha}\right),
$$

1 P. Lévy, op. cit., p. 167.

${ }^{2}$ P. Lévy, op. cit., p. 171.

${ }^{3}$ There exists a derivative $\sigma^{\prime}(x)$ up to a set of measure zero even if $\sigma(x)$ is not absolutely continuous, but

$$
\sigma(x)=\int_{-\infty}^{x} \sigma^{\prime}(y) d y
$$

holds if and only if $\sigma(x)$ is absolutely continuous. It is meaningless to regard $\sigma(x)$ as a density of probability if (i) is not, valid. 
where $a>0$ may be arbitrarily small, and there exist ${ }^{1}$ symmetric distribution functions which satisfy (5b) but are not absolutely continuous. Conversely, the absolute continuity of $\sigma$ does not imply (5) since the Riemann-Lebesgue lemma cannot be formulated by using a universal majorant which tends to zero. A sufficient condition for (5b), hence for (5) and (5a), is that there exist a density of probability satisfying a uniform Lipschitz condition of arbitrarily low index, or only the corresponding logarithmical estimate, and tending not too slowly to zero as $x \rightarrow \infty$. Another sufficient condition for (5) and (5a) is that $\sigma$ satisfy the Gauss postulate for error distributions, i.e., that there exist for every $x$ a probability density which does not increase when $x$ increases. In fact, in this case it is clear from (3), in virtue of the second mean-value theorem, that $L(t ; \sigma)=O\left(t^{-1}\right)$, so that $(5 b)$ is amply satisfied.

Let the random variables $x_{1}, x_{2}, \ldots, x_{k}, \ldots$ be such that $\sigma(x)$ represents the probability of the inequality $x_{k}<x$ for every $k$. Then if $\sigma_{n}(x)$ denotes the probability of the inequality $x_{1}+x_{2}+x_{3}+\ldots+x_{n}<x$, we have

$$
L\left(t ; \sigma_{n}\right)=L(t ; \sigma)^{n}
$$

in virtue of the supposed independence of the random variables ${ }^{2}$. The fundamental limit theorem of the calculus of probability ${ }^{3}$ implies that the distribution function $\sigma\left(a_{n} x\right)$, where

$$
a_{n}=M_{2}\left(\sigma_{n}\right)^{\frac{1}{2}}=n^{\frac{1}{2}} M_{2}^{\frac{1}{2}}=n^{\frac{1}{2}}
$$

tends, as $n \rightarrow+\infty$, to the reduced Gaussian distribution function. Our purpose is to show that $\sigma_{n}(x)$ is capable of an infinite asymptotic development in the Poincaré sense, proceeding according to powers of $n^{-\frac{1}{3}}$. The rôle of assumption (5) is that of assuring the existence of such a development, formal treatments of which date back to Laplace. The coefficient of $\left(n^{-\frac{1}{3}}\right)^{m}$ in the asymptotic series in question is a polynomial in $x$ having as coefficients polynomials in the moments (1) of the elementary law $\sigma$, and the coefficients of the

${ }^{1}$ Cf. D. Menchoff, "Sur l'unicité du développement trigonométrique," Comptes Rendus, 163 (1916), pp. 433-436.

2 Of., e.g., P. Lévy, op. cit., pp. 184-185.

3 Ibid, pp. 233-235.

4 Of., e.g., E. T. Whittaker and G. Robinson, The Calculus of Observations, London, 1924, p. 172 ; cf. also F. Zernike, Handbuch der Physik, 3 (1928) 450-51, where further references are also given. 
latter polynomials are universal constants. The elementary laws occurring in the majority of applications satisfy (5) and are such that the Carleman condition

$$
\sum_{m=0}^{+\infty} M_{2 m}^{-1 /(2 m)}=+\infty
$$

of determinateness is fulfilled. Hence we obtain a method, at least in theory, for determining the elementary law $\sigma(x)$ from the behaviour of the approximation of the iterated law to the Gauss distribution.

The function $L(t ; \sigma)$ has for every $t$ derivatives of arbitrarily high order ${ }^{1}$ which may be obtained by formal differentiation of (3), so that

$$
L^{(m)}(t ; \sigma)=i^{m} \int_{-\infty}^{+\infty} x^{m} e^{i t x} d \sigma(x) .
$$

In fact, each of the integrals (7) is uniformly convergent with respect to $t$, its integrand having as a majorant ${ }^{2}$ that of $M_{m}$.

It is clear from (3) that $|L(t ; \sigma)| \leqq 1$ for every $t$ and $=1$ for $t=0$. Suppose that $|L(t ; \sigma)|=1$ for a fixed $t$. Then

$$
\int_{0}^{+\infty}\{1 \pm \cos (t x)\} d \sigma(x)=0,
$$

where $1 \pm \cos (t x) \geqq 0$ for every $x$ and either $t=0$ or else $1 \pm \cos (t x)>0$ for some $x$. Hence either $t=0$ or else $\sigma(x)$ is a step-function having all its jumps at points $x$ which form an arithmetical progression. The second case is excluded, $\sigma$ being continuous in virtue of (5). Consequently ${ }^{3}$

$$
|L(t ; \sigma)|<1 \text { for every } t \neq 0 .
$$

Moreover, since the second derivative of (3) is negative at $t=0$ in virtue of (7), and the first derivative $L^{\prime}(t ; \sigma)$ vanishes at $t=0$ because of (7) and (2), we have $L^{\prime}(t ; \sigma)<0$ for sufficiently small values of $t>0$. It follows therefore from $L(0 ; \sigma)=1$ that $L(t ; \sigma)$ is positive and decreasing in the interval $0<t \leqq c$ if $c$ is sufficiently small. Let $c$ be so chosen and put

$$
K_{n}=\int_{c}^{+\infty}\left|t^{-1} L(t ; \sigma)^{n}\right| d t
$$

1 It is not true, however, that $L(t ; \sigma)$ is necessarily regular-analytic along the $t$-axis.

2 In virtue of the Schwarz inequality it is sufficient to consider even values of $m$.

${ }^{3}$ It may be mentioned that (8) is actually false in the second case. In fact, $L(t ; \sigma)$ is then a periodic function so that $L(t ; \sigma)=1$ holds for some $t \neq 0$ since it holds for $t=0$. 
Now $L(t ; \sigma)$ has in the interval $c \leqq t<+\infty$ a positive maximum $\theta<1$ according to (8) and (5a). On the other hand, it is clear from (5) and (9) that $K_{j}<+\infty$ if $j$ is sufficiently large, so that $K_{n}<\theta^{n-j} K_{j}<+\infty$ for every $n>j$. Consequently

$$
\left|\int_{c}^{+\infty} L(t ; \sigma)^{n} t^{-1} \sin (t x) d t\right|<C \theta^{n} \text {, where } 0<\theta<1,
$$

for every $x$ and for every $n>j$, where $\theta$ and $C=K_{j} / \theta^{j}$ depend only upon $c$.

Since $L(0 ; \sigma)=1$ and $L(t ; \sigma)$ is positive and decreasing in the range $0<t \leqq c$, the function

$$
s=s(t)=\{-\log L(t ; \sigma)\}^{t}
$$

is positive and increasing in this range so that there exists an inverse function $t=t(s)$, where $0 \leqq s \leqq d$ and $d=\{-\log L(c ; \sigma)\}^{\ddagger}$. Now the derivative $L^{\prime}(t ; \sigma)$ is negative at every point of the range $0<t \leqq c$ and vanishes at $t=0$ only in the first order in virtue of $L^{\prime \prime}(0 ; \sigma)=-1$; hence the function $s=s(t)$ vanishes at $t=0$ exactly in the first order, and consequently $r(t)=t / s(t)$ is positive at $t=0$. Upon placing, for a fixed value of $x$,

$$
\pi f(x ; s)=\sin (x t(s)) \dot{t}(s) / t(s) \quad(0 \leqq s \leqq d),
$$

where the dot denotes differentiation with respect to $s$, it follows from the Bürmann-Lagrange rule ${ }^{1}$ that all derivatives of $f(x ; s)$ with respect to $s$ exist not only in the range $0<s \leqq d$, but at $s=0$ as well, and, moreover, that the derivatives are given by the explicit formula

Setting

$$
\left\{\frac{\partial^{n} f(x ; s)}{\partial s^{n}}\right\}_{s=0}=\frac{1}{\pi}\left\{\frac{\partial^{n}}{\partial t^{n}} \frac{\sin (t x) r(t)^{n+1}}{t}\right\}_{t=0} .
$$

$$
\chi_{n}(x)=\frac{1}{2}+\frac{1}{\pi} \int_{0}^{c} L(t ; \sigma)^{n} t^{-1} \sin (t x) d t,
$$

we have from (11) and (12)

$$
\chi_{n}(x)=\frac{1}{2}+\int_{0}^{d} \exp \left(-n s^{2}\right) f(x ; s) d s .
$$

This function $\chi_{n}(x)$ admits for every fixed $x$ an asymptotic development ${ }^{2}$

$$
\frac{1}{2}+\sum_{k=1}^{+\infty} P_{k}(x) n^{-t k}
$$

${ }^{1}$ Of. P. L. Tchebychef, Oeuvres, vol. 1, St. Pétersburg, 1899, pp. 251-270, where analyticity of the functions is not required.

${ }^{2} \mathrm{Cf}$. A. Wintner, "On the asymptotic formulae of Riemann and of Laplace," Proceedings of the National Academy of Sciences, 20 (1934), pp. 57-62. 
where

or

$$
P_{k}=P_{k}(x)=\frac{\Gamma\left(\frac{1}{2} k\right)}{2 \Gamma(k)}\left\{\frac{\partial^{k-1} f(x ; s)}{\partial s^{k-1}}\right\}_{s=0}
$$

Thus

(18) $\quad P_{2 k+1}=\frac{\Gamma\left(k+\frac{1}{2}\right)}{2 \pi \Gamma(2 k+1)} \sum_{\nu=0}^{k}\left(\begin{array}{l}2 k \\ 2 \nu\end{array}\right)(-1)^{k-\nu} \frac{x^{2(k-\nu)+1}}{2(k-\nu)+1}\left\{\frac{d^{2 \nu} r(t)^{2 k+1}}{d t^{2 \nu}}\right\}_{t=0}$

by the Leibniz rule for differentiation, while $P_{2 k}=0$ for every $x$ because of (2). In particular

$P_{1}(x)=(2 \pi)^{-\frac{1}{3}} x$,

$P_{3}(x)=(2 \pi)^{-\frac{1}{2}}\left\{-\frac{x_{3}}{6}+\left(M_{4}-3\right) \frac{x}{8}\right\}$,

$P_{5}(x)=(2 \pi)^{-\frac{1}{2}}\left\{\frac{x^{5}}{40}-5\left(M_{4}-3\right) \frac{x^{3}}{48}+\left(35 M_{4}^{2}-8 M_{6}-90 M_{4}+75\right) \frac{x}{384}\right\}$.

It is clear from (10) that the above asymptotic expansion of (14) is also an asymptotic development of

$$
\frac{1}{2}+\frac{1}{\pi} \int_{0}^{+\infty} L(t ; \sigma)^{n} t^{-1} \sin (t x) d t .
$$

Moreover, upon applying (4) to $\sigma_{n}(x)$ instead of $\sigma(x)$, we see from (6) that (19) is exactly $\sigma_{n}(x)$. Therefore (15) is an asymptotic development of $\sigma_{n}(x)$.

It may be mentioned that (15) can in certain cases be a convergent series. For example, if $\sigma(x)$ obey the Gauss law the asymptotic development (15) for $\sigma_{n}(x)$ is found from (18) to be the convergent power-series representation of $\sigma_{n}(x)$. 